\title{
Cyclooxygenase-2 (COX-2): a molecular target in prostate cancer
}

\author{
G. Aparicio Gallego a , S. Díaz Prado ${ }^{\mathrm{a}}$, P. Jiménez Fonseca ${ }^{\mathrm{b}}$, R. García Campelo ${ }^{\mathrm{c}}, \mathrm{J}$. \\ Cassinello Espinosa ${ }^{\mathrm{d}}$ and L.M. Antón Aparicio ${ }^{\mathrm{c}, \mathrm{e}}$
}

\author{
${ }^{a}$ Oncology Research Unit. CHU Juan Canalejo. A Coruña, Spain \\ ${ }^{b}$ Medical Oncology Service. Central Hospital. Asturias. Oviedo, Spain \\ ${ }^{c}$ Medical Oncology Service. CHU Juan Canalejo. A Coruña, Spain \\ ${ }^{d}$ Medical Oncology Service. Guadalajara University Hospital. Guadalajara, Spain \\ ${ }^{e}$ Department of Medicine. University of A Coruña. A Coruña, Spain
}

\begin{abstract}
Epidemiological studies provided the first evidence that COX may be involved in the pathogenesis of cancer. In the process of carcinogenesis and in the route of intracellular signalling during carcinogenesis, COX-2 expression may be a universal phenomenon. In general, COX-2 is up-regulated throughout the tumorigenic process, from early hyperplasia to metastatic disease. COX-2 has been reported to be constitutively overexpressed in a variety of malignancies and is frequently constitutively elevated in prostate carcinoma. COX-2 was consistently overexpressed in premalignant lesions such as prostatic intraepithelial neoplasia, and carcinoma. Cases are described with evolution of proliferative inflammatory atrophy of the prostate and prostate carcinoma. The increase of evidence implicating COX-2 in cancer has stimulated clinical trials to investigate the efficacy of selective COX-2 inhibitors in individuals at risk for human cancer. Regarding prostate carcinoma there is much direct or indirect evidence to support the use of COX-2 inhibitors in this disease. Trials using these drugs in familial adenomatous polyposis (FAP) and other patients with a high risk of colorectal carcinoma are ongoing.
\end{abstract}

Key words

Prostate cancer; Cyclooxygenase-2; Inhibitors; Pharmacological treatment; Molecular target 


\section{Introduction}

COX-1 and COX-2 are the two isoforms of cyclooxygenase, which convert arachidonic acid (AA) into several eicosanoids such as prostaglandin, thromboxanes and prostacyclin, which participate in several normal physiologic processes and inflammation. Whereas COX-1 is constitutively expressed in most tissues, COX-2 is an inducible enzyme, stimulated by cytokines, growth factors, oncogenes or tumour promoters during inflammation or malignancy. At present, the overexpression pattern of COX-2 in prostate carcinoma is unresolved. While some reports suggest no significant increase in COX-2 expression in prostate tumour tissue when com- pared to normal tissues, others have reported a direct association of prostate tumour grade and poorly differentiated prostate cancer with COX-2 levels.

Both autocrine and paracrine mechanisms may be responsible for the development and/or progression of androgen-independent prostate cancer either directly by stimulation of tumour cell proliferation or indirectly by enhanced prostaglandin-regulated local androgen synthesis.

One of the first clues that cyclooxygenase inhibition might be an effective approach to preventing cancer came from epidemiological studies. The fact that all non-steroidal anti-inflammatory drugs (NSAIDs) in clinical use are cyclooxygenase inhibitors provides a putative link between the inhibition of COX activity and the antitumour effect of NSAIDs.

The underlying hypothesis examined is whether COX-2 inhibition would enhance response to cytotoxic chemotherapy through inhibition of tumour angiogenesis, promotion of apoptosis or other alternative mechanisms.

\section{COX-2 and prostate carcinoma}

The molecular pathogenesis of prostate cancer has been characterised by alterations of genes and proteins involved in proinflammatory pathways. Earlier studies have indicated that one of the prostate cancer precursor lesions, proliferative inflammatory atrophy (PIA), shares some molecular traits with prostatic intraepithelial neoplasia (PIN), and both of these two early precursor lesions of the prostate promote further cancer growth. At this juncture, it is worth recalling the findings from earlier studies showing a strong correlation between the levels of AA metabolites and accumulation of proinflammatory prostaglandins in prostate carcinogenesis.

COX-2 has been reported to be constitutively over- expressed in a variety of malignancies and is frequently constitutively elevated in prostate carcinoma. COX-2 appears to be induced in prostate adenocarcinoma cells in dogs [1].

Numerous investigators have evaluated the expression of COX-2 in human prostate cancer with conflicting results. Several studies have suggested that COX-2 is commonly overexpressed in prostate cancer [2-5]. In contrast, others have found that levels of COX-2 are extremely low or absent in the majority of prostate cancers $[6,7]$.

Several lines of evidence implicate 12-lipoxygenase (12-LOX) as a regulator of human prostate cancer [8], and are expressed in the prostate cancer cell lines DU- 145 and PC3 [9]. Platelet-type 12-LOX has been shown to regulate growth, metastasis, and angiogenesis of prostate cancer: the 12-LOX pathway is a critical regulator of prostate cancer progression and apoptosis, by affecting various proteins regulating these processes.

Platelet-type 12-LOX expression was detected in human prostatic tumours and correlated to the clinical stage of disease. Human prostate cancer cell lines express the platelet-type isoform of 12-LOX at both the mRNA and protein levels, and immunohistochemistry revealed 12-LOX expression in human prostate tumours. The enzyme was localised to the plasma mem- brane, cytoplasmic organelles and nucleus in non- metastatic cells (PC-3 nm) and to the cytoskeleton and nucleus in metastatic cells (DU145) [9]. 
A study involving the human prostate cancer cell lines PC-3 nm and $\mathrm{LNCaP}$ demonstrated upregulated COX-2 mRNA expression and increased cell proliferation, total DNA content and endogenous prostaglandin $\mathrm{E}_{2}\left(\mathrm{PGE}_{2}\right)$ production in response to the administration of exogenous dimethylprostaglandin E2 [10]. $\mathrm{PGE}_{2}$, as a downstream metabolite of linoleic acid (LA), can increase cell growth as well as the enzyme responsible for its own synthesis. Growth of the androgen-unresponsive PC-3 nm human prostate cancer cells is stimulated in vitro by the addition of the omega- 6 polyunsaturated LA. The growth effects of essential fatty acids appear to involve both PGs and leukotrienes, which interconnect with autocrine regulation through epidermal growth factor (EGF)-related polypeptides. Studies in many laboratories have indicated that the fatty acid composition of the diet, as well as the absolute amounts of fat consumed, may influence prostate cancer risk. Prostate cancer incidence rates have been increasing and arguments have been postulated that there might be a relationship between the rising cancer risk and an increase in the consumption of vegetable oils rich in LA.

As a first approach to the question, a series of experiments in vitro are performed to examine the effects of both LA, an n-6 polyunsaturated fatty acid (PUFA), and docosahexaenoic acid (DHA) and eicosapentaenoic acid (EPA), two n-3 fatty acids, on the growth of human prostate cancer cells [11]. The n-3 fatty acids compete with LA and its metabolic product AA for enzymes that regulate eicosanoid biosynthesis. When AA is formed from LA, it is largely incorporated into the cell membrane phospholipids where it provides a reserve of substrate for eicosanoid biosynthesis. Mobilisation of AA involves phospholipase A2, an enzyme that has been shown to be stimulated by EGF [12, 13] (Fig. 1). In addition, EGFR has been proposed to be localised with PI3K and ceramide molecules within the plasma mem- brane microdomains (caveolae/raft structures), which are enriched in sphingolipids and cholesterol $[14,15]$.

In vitro, like PC-3 nm cells, the DU145 human prostate cancer cell line does not require the presence of androgens for its growth; it grows readily in the absence of serum and synthesises both EGF and the related polypeptide transforming growth factor $\alpha$ (TGF- $\alpha$ ). Both PC-3 nm and DU145 cells possess EGF/TFG- $\alpha$ receptors, and it has been suggested that an autocrine loop exists for the regulation of prostate cancer cell growth [11] (Fig. 1).

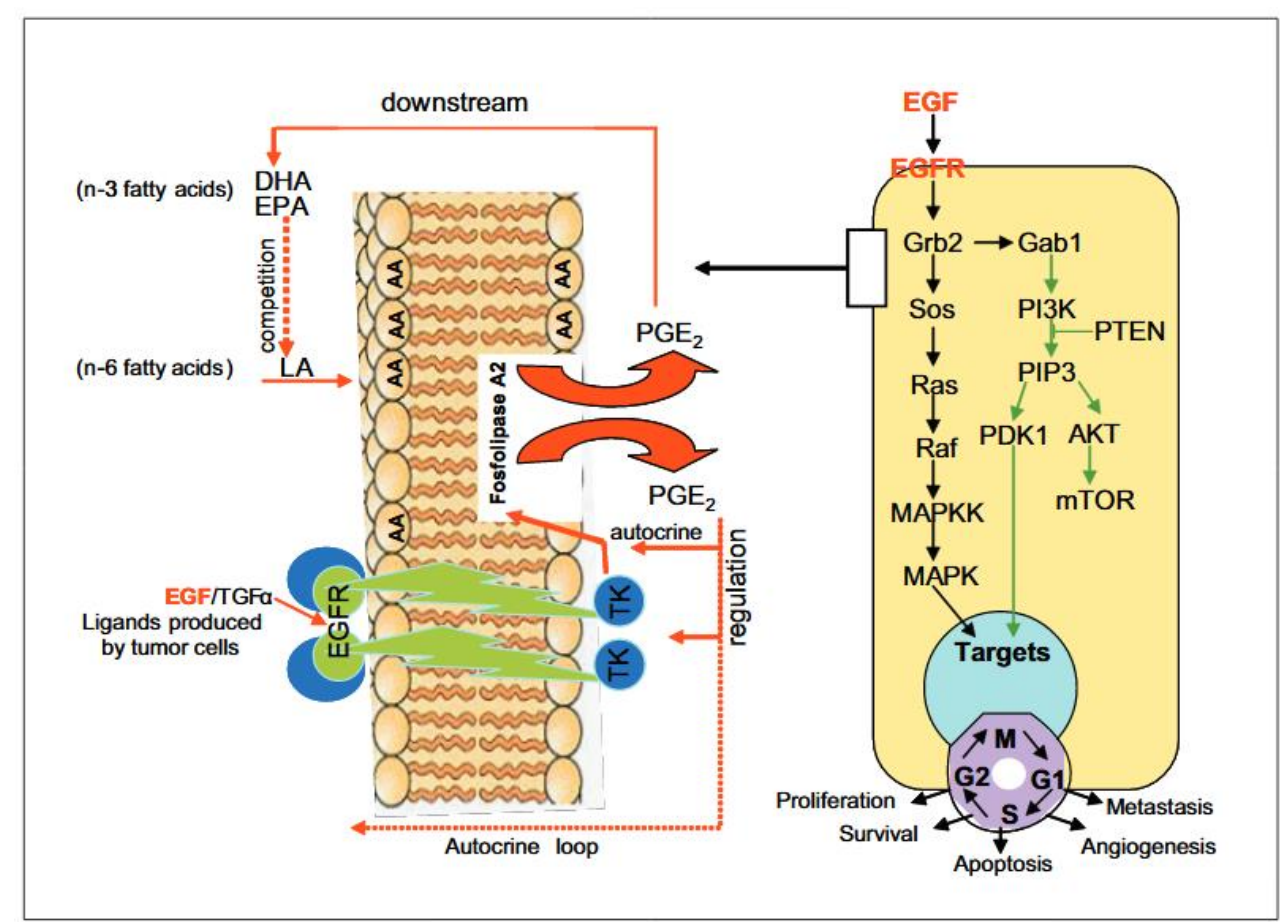

Fig. 1. Interaction of n-6 fatty acid (LA) and EGF in eicosanoid biosynthesis. DHA and EPA, both n-3 fatty acids, compete with LA and its metabolic product AA. Mobilisation of AA involves phospholipase A2, an enzyme that stimulates $\mathrm{PGE}_{2}$ synthesis and which has been shown to be stimulated by EGF 
The expression of COX-1 is found in prostate tissues as well as in prostate carcinoma samples [16]. The expression of COX-2 was modulated by the degree of adenocarcinoma differentiation (Fig. 2): in the low and middle groups of adenocarcinoma the expression was strong and there was a very strong expression level in the high group of adenocarcinomas. The expression of COX-2 also was high in the blood vessels and stromal tissues of prostate carcinoma samples. However, the expressions of COX-2 in the blood vessels and stromal tis- sues from benign prostatic hypertrophy, PIN and normal prostate samples were at basic levels [16]

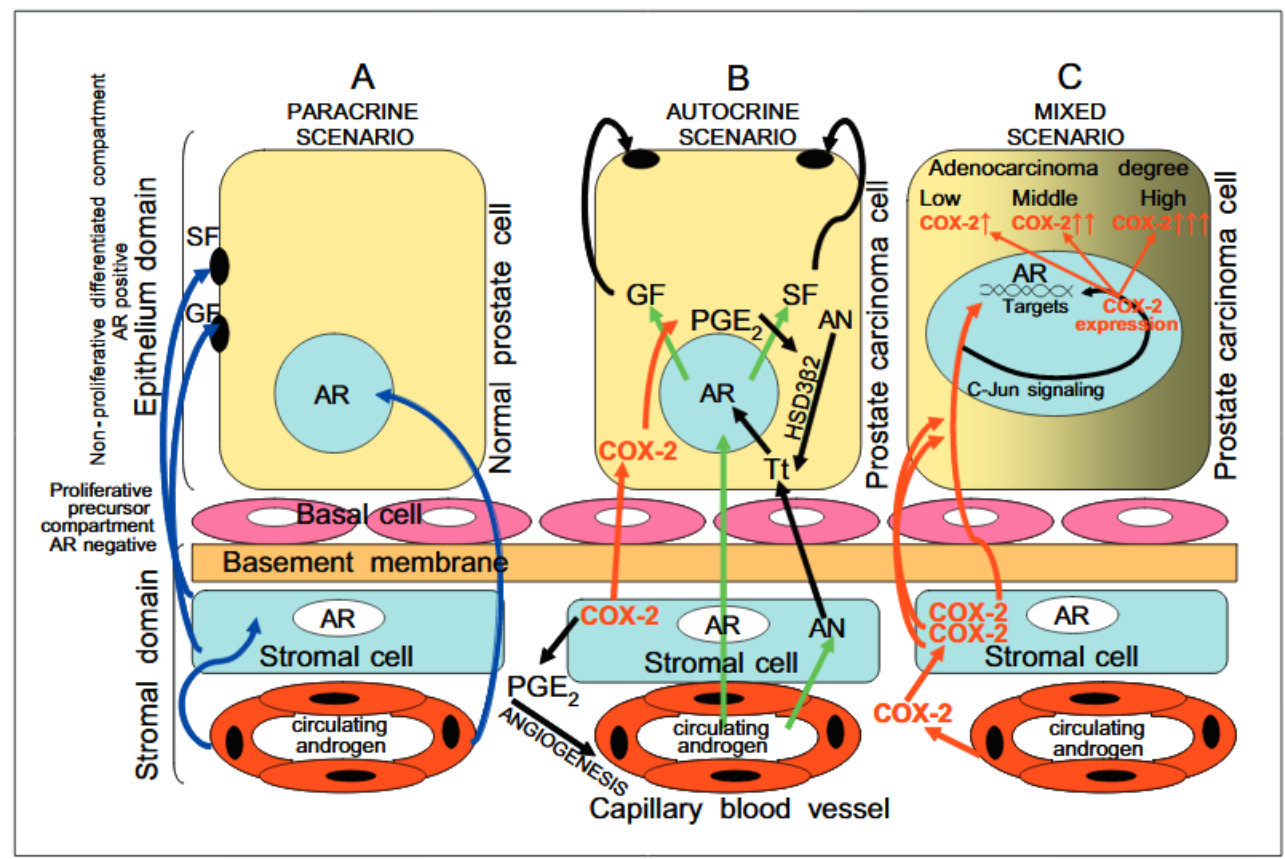

Fig. 2A. In normal prostate gland, growth and maintenance of prostate epithelium depends on growth factors (GF) and survival factors (SF) produced by stromal cells. GF and SF are produced as a result of androgen signalling through AR. B. During transformation to cancer, the paracrine mechanism of AR action is replaced by an emergent autocrine mechanism, whereby cancer cells are less dependent on stromal cell factors. Hypothetical COX-2-derived $\mathrm{PGE}_{2}$ potentiates androgen biosynthesis by the $3 \beta$-hydroxysteroid dehydrogenase type 2 (HSD3 32 ) enzyme and promotes angiogenesis, cell proliferation survival and metastasis. $A N$, androstenedione; $T t$, testosterone. C. Both autocrine and paracrine mechanisms may be responsible for the development and/or progression of androgen-independent prostate cancer by enhanced prostaglandin-regulated local androgen synthesis. COX-2 is a potent stimulator of AR function and the induction of c-Jun expression/phosphorylation plays an important role. C-jun exhibits an inhibitory effect on AR protein activity; c-Jun can inhibit the transcription activities of the AR promoter

Moreover, elevated 12-LOX mRNA levels were detected in cancer cells and the expression associated with poor differentiation and invasiveness of prostate cancer: approximately one-third of patients showed elevated levels of 12-LOX mRNA in prostate cancer tissues com- pared with the matching normal tissues [12]. The in- crease of 12-LOX expression in prostate cancer was found to correlate with advanced stage, poor differentiation and invasive potential. More importantly, elevation in the expression of this marker in prostate cancer patients may predict the disease aggressiveness in this sub- set of patients [17].

The immunohistochemical analysis of human prostate carcinoma reveals that COX-2 expression is multi- focal and moderate to strong in the majority of cases. Staining for COX-2 is mild in benign tissue as com- pared to prostate carcinoma tissue. Expression of COX- 2 in prostate carcinoma tissue is predominantly granular and mostly localised in tumour cells. Interestingly, COX-2 expression in prostate carcinoma tissues is greater in the surrounding stromal cells or infiltrating inflammatory cells than in tumour cells. This observation raises interesting possibilities about the localisation of COX-2-expressing cells in prostate carcinoma. Therefore, stromally derived COX-2 may promote tumour growth by producing bioactive prostaglandins which affect carcinoma cells in a paracrine fashion. COX-2 may thus be acting as a "landscaping tumour promoter" according to the landscaping model [18]. 
Tumour cell invasion and metastasis are complex processes involving multiple host-tumour interactions (stromal-epithelium). [12(S)-Hydroxy eicosatetraenoic acid] [12(S)-HETE] can enhance the invasive and metastatic potentials of tumour cells by modulating several steps of this complex process. There is evidence to suggest that the ability of tumour cells to generate 12(S)-HETE is correlated with their metastatic potential [19]. A growing body of recent evidence suggests that 12(S)-HETE may act as a second messenger in stimulus-response coupling in some cells.

There are conflicting data regarding whether COX-2 is increased in the epithelial or in the stromal component of tumours. COX-2 has been found to be increased in the epithelium of adenomas (i.e., colonic epithelium). It has been found that COX-2 expression is increased in the stromal component of adenomas $[20,21]$.

\section{Mechanisms of cyclooxygenase inhibitors}

The molecular mechanisms by which NSAIDs exhibit anticancer activities are not completely understood (Table 1) and it is plausible that COX-2-inhibitor-mediated apoptosis works through both COX-2-activity-dependent and -independent pathways.

Table 1. The molecular mechanisms of cyclooxygenase inhibitor (celecoxib) at cellular levels via COX-2 independent mechanisms against prostate cancer

The molecular mechanisms of celecoxib

- Caused an increase in the number of cells at the $\mathrm{G}_{1}-\mathrm{S}$-phase transition point with a trend toward accumulation of more cells in the $\mathrm{G}_{1}$ phase and a corresponding decrease in the percentage of cells in the $\mathrm{S}$ phase $[29,30]$.

- Induces cell cycle arrest at the $\mathrm{G}_{1}-\mathrm{S}$-phase transition point and modifies cell cycle regulatory proteins such as cyclin D1, retinoblastoma $(\mathrm{Rb})$, phosphorylated $\mathrm{Rb}$, Cyclin E, p2 $7^{\mathrm{KIP1}}$ and $\mathrm{p} 21^{\mathrm{WAF} 1 / \mathrm{CIP1}}[74]$.

- Perturb intracellular calcium by inhibiting endoplasmic reticulum $\mathrm{Ca}^{2+}$-ATPases [75].

- Blocks Akt signalling by inhibiting PDK-1 kinase activity [25-29].

- Enhanced expression and phosphorylation of c-Jun in LNCaP cells. Overexpression of c-Jun inhibited the AR promoter [33].

- Inhibited the expression of androgen up-regulated genes [33].

- Inhibited AR-induced gene expression and AR promoter activity at the transcription level [36]

- Overexpressed c-Jun by the NSAIDs not only inhibited the function of AR but also directly repressed AR expression at the transcription level [34-37].

- Induced apoptosis in prostate cancer cells is caspase-3 and -9 mediated [63].

- Induced apoptosis was associated with down-regulation of COX-2, nuclear factor $\mathrm{kBp} 65$, and with activation of peroxisome proliferator-activated receptor ã, apoptosis activating factor-1 and caspase-3 [31, 32].

- Inhibited cancer cell growth and mediated protein-protein interactions between heat shock protein 70 (HSP70) and p53 at low doses [76].

The biochemical mechanism underlying COX-2-inhibitor-induced apoptosis, however, remains elusive. At least three distinct mechanisms have been proposed: first, NSAID treatment of cells resulted in an increase in AA that, in turn, stimulated the production of ceramide, a known death signal, by activating neutral sphingomyelinase [22]; second, the COX-2 inhibitors have been reported to sensitise prostate cancer cells to apoptosis by down- regulating the anti-apoptotic protein Bcl-2 [23, 24]; third, a study shows the involvement of a COX-2-independent pathway during the apoptosis signalling [25].

Celecoxib induces apoptosis, in part, by blocking the activation of the antiapoptotic kinase Akt (also called protein kinase B). The evidence from laboratories has demonstrated the involvement of molecular targets other than COX-2 in celecoxib-mediated in vitro antiproliferative effects in prostate [25-28]; the 3-phosphoinosi- tide-dependent protein kinase-1 (PDK-1)/Akt signalling pathway represents a major COX-2-independent mechanism by which celecoxib exerts in vivo antiproliferative effects against prostate tumours [29]. Among various putative pathways reported in the literature, blockade of Akt signalling is especially noteworthy [26-28]. 
It is well documented that PDK-1/Akt signalling plays a central role in regulating cancer cell survival and proliferation by affecting the phosphorylation status of a plethora of downstream effectors that are either PDK-1 or Akt substrates [30]. Consequently, blockade of this signalling cascade leads to growth inhibition by cell cycle arrest and/or apoptosis in malignant cells.

Moreover, celecoxib inhibits adenocarcinoma of the transgenic adenocarcinoma of mouse prostate (TRAMP) in a dose-dependent manner, and thus limits the growth of metastatic prostate cancer: immunohistochemical detection of proinflammatory protein, NF- $\kappa$ Bp65 and COX-2 indicate downregulation by celecoxib. Suppression of the above proinflammatory proteins was associated with a higher rate of apoptosis and reduced tumour growth [31].

Earlier studies on immunohistochemical detection of NF- $\kappa$ in tissues from human prostatectomy demonstrated overexpression of active subunit of NF- $\kappa$ Bp65 [32].

Observations strongly indicate a correlation in the down-regulation of COX-2 with targets such as NF- $\kappa$ Bp65 that makes cells susceptible to the actions of apoptosis-inducing mechanisms that are believed to be simultaneously activated by celecoxib.

In the other way, some reports strongly suggest that NSAIDs are potent inhibitors of androgen receptor (AR) function and that the induction of c-Jun expression/phosphorylation [33] plays an important role in mediating their effects. Previous studies [34-37] demonstrated that c-Jun exhibits an inhibitory effect on AR protein activity. In addition, c-Jun can inhibit the transcription activities of the AR promoter [33]. c-Jun is a component of AP-1 protein complex and a member of the basic leucine zipper family of sequence-specific dimeric DNA-binding proteins. Stimulated overexpression of c- Jun protein can inhibit the function of the AR [34-37]. The transactivation functions of the AR as well as other steroid receptors can be affected by c-Jun. The interaction between the AR and c-Jun is mediated through the DNA- and ligand-binding domains of AR and the leucine zipper region of c-Jun that may affect transcriptional function of the AR [36]. Other studies indicate that the transcriptional interference between AR and c- Jun is partly mediated through competition for intracellular cAMP-responsive element binding protein [37], which serves as an integrator/coactivator for AR trans- activation activities.

\section{Clinical prospects and future directions for COX-2 inhibitors}

The increasing evidence implicating COX-2 in cancer has stimulated clinical trials to investigate the efficacy of selective COX-2 inhibitors in individuals at risk for human cancer. Treatment with COX-2 inhibitors has been shown to reduce the size and number of polyps in familial adenomatous polyposis (FAP) patients, and is currently being evaluated for efficacy in preventing sporadic colorectal adenomas $[38,39]$. There is enough reviewed evidence that aberrant COX-2 expression is also associated with breast cancer: selective COX-2 inhibitors have proved effective in preventing experimental breast cancer [40].

Some, but not all, studies suggest that COX-2 is overexpressed in primary human prostate cancers and in human prostate cancer cell lines [41-45]. Selective COX-2 inhibitors induce apoptosis of human prostate cancer cells in vitro and in vivo.

Attending to prostate carcinoma, there is much direct or indirect evidence to support the use of COX-2 inhibitors in this disease. Inhibition of cyclooxygenase by traditional NSAIDs and other selective inhibitors of the proinflammatory COX-2 isoform may also prove to have chemopreventive efficacy in prostate.

The results of case-control studies suggest that men who regularly took NSAIDs may have a reduced relative risk of prostate cancer, particularly for advanced cases of the disease [46]. Limited epidemiological studies suggest that NSAID use is associated with reduced risk of prostate cancer [4648]. Elevated levels of $\mathrm{PGE}_{2}$ and $\mathrm{AA}$ metabolism are seen in benign and malignant human prostate tumour cells, and NSAIDs inhibit prostate tumour cell growth [49-52]. 
Two NSAID derivatives that have lost COX inhibitory but retained pro-apoptotic activity are also promising chemopreventive agents in prostate: the sulphone metabolite of sulindac most probably stimulates apoptosis via inhibition of cyclic GMP phosphodiesterase. This drug was found to inhibit the rise of (prostate-specific antigen) PSA in patients with recurring prostate cancer after radical prostatectomy [53].

In addition, it has also been shown that the 12-LOX pathway of AA metabolism regulates cell growth, through inhibition of cell proliferation, protein regulating apoptosis, transition in the cell cycle and $\mathrm{Rb}$ protein family members. Inhibition of 12-LOX led to growth inhibition associated with a specific G1 arrest, followed by induction of apoptosis through caspase and Bcl-mediated mechanisms [54].

As COX-2 is overexpressed in HER-2/neu-positive breast cancer, selective COX-2 inhibitors should be evaluated as therapy in the prostate cancer population. Because COX-2-derived PGs may enhance aromatase activity, a therapeutic regimen combining a selective COX-2 inhibitor with an aromatase inhibitor should be considered. Actually, in some hormone-resistant prostate tumours, glucocorticoid was added to complement the treatment with good success. Hormonal therapy with glucocorticoid may constitute a basic or first treatment for some prostate carcinoma tumours in patients with an adenocarcinoma that has a Gleason score $\geq 8$ by including glucocorticoid therapy as soon as the diagnosis is confirmed. Transcription of COX-2 is inhibited markedly by glucocorticoid therapy.

NSAIDs have been seen to inhibit NF- $\kappa$ B signalling (Fig. 3) by directly blocking the activity of I $\kappa \mathrm{B}$ kinase $\beta[55,56]$. Additionally, indomethacin, a non- selective COX inhibitor, can act as a direct ligand for peroxisome proliferator activating receptor (PPAR) $\alpha$ and $\gamma$, and thus may directly regulate gene transcription [57]. The ability of PPARs to bind eicosanoids suggested that PPAR $\delta$ might be a target of chemopreventive nonsteroidal anti-inflammatory drugs [58-60]. The findings suggest that PPAR $\delta$ represents a $\beta$-catenin/Tcf- 4 target with particular importance for chemoprevention. Whereas APC or $\beta$ catenin mutations can result in increased PPAR $\delta$ activity, NSAIDs can compensate for this defect by suppressing PPAR $\delta$ activity and promoting apoptosis. This suppression of PPAR $\delta$ is mediated in part by the ability of some NSAIDs to directly inhibit the DNA-binding activity of PPAR $\delta$ (Figs. 3 and 4 ).

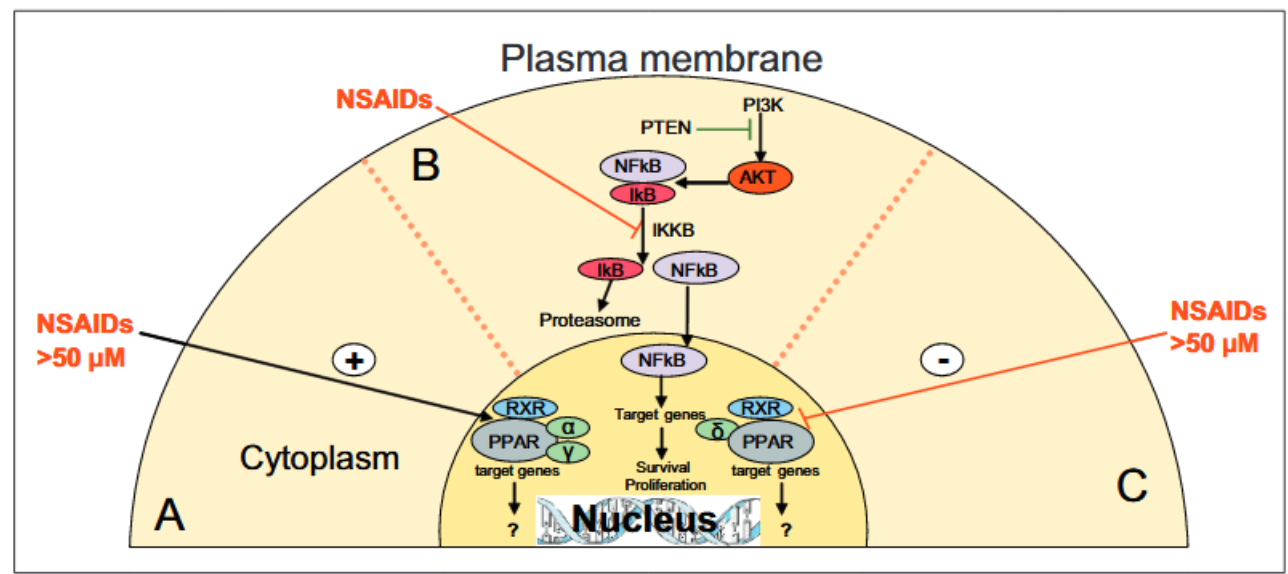

Fig. 3A. There is evidence that PGs can modulate cellular pathways by acting directly within the nucleus. Certain NSAIDs can activate peroxisome proliferator activated receptor subtypes (PPAR $\alpha$ and PPAR $\gamma$ ). B. NSAIDs can inhibit the activity of IkB kinase $\beta$ (IKK $\beta$ ); this leads to antagonism of the NF- $\kappa$ B signalling pathway by blocking the destruction of the NF- $\mathrm{BB}$ inhibitor $\mathrm{IkB}$, preventing NF- $\mathrm{BB}$ from translocating to the nucleus and regulating key target genes. C. Other NSAIDs have been shown to inhibit the DNA-binding activity of peroxisome proliferator activated receptor subtype (sulindac) 


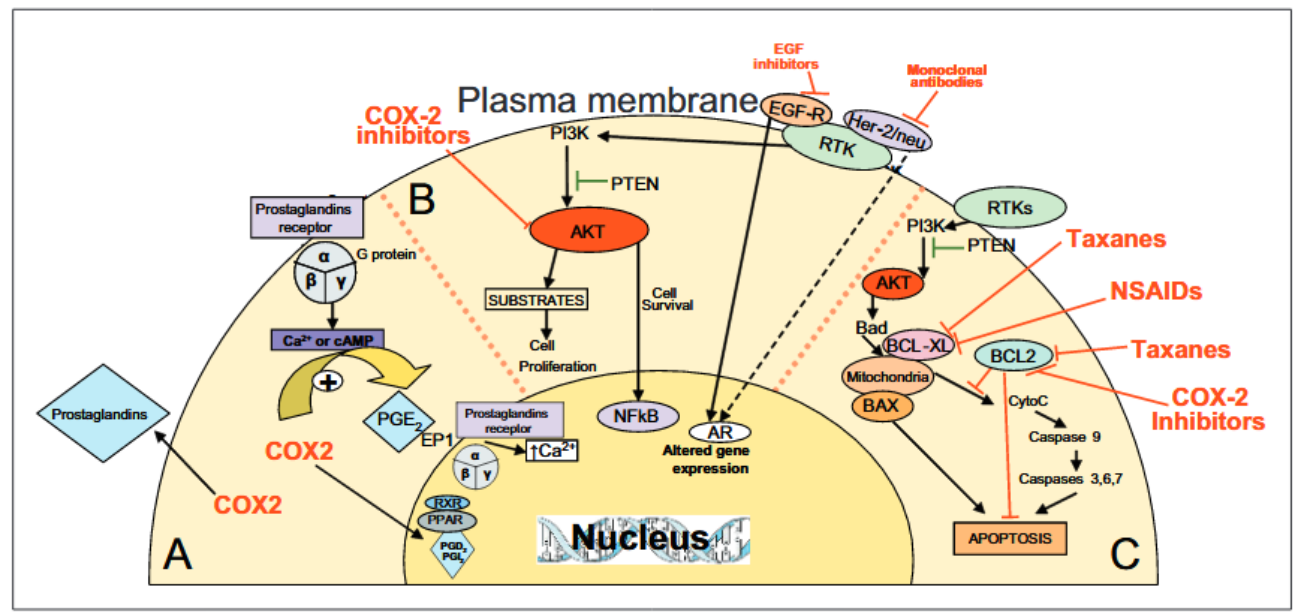

\begin{abstract}
Fig. 4A. The different classes of PGs exert their effects by binding to a G-protein-coupled cell-surface receptor leading to changes in the cellular levels of cAMP and $\mathrm{Ca}^{2+}$, or by acting directly within the nucleus. PGE 2 works by its receptor EP1 (localised to the nuclear envelope) and activation of the receptor leads to change in nuclear levels of $\mathrm{Ca}^{2+}$, retinoid X receptor (RXR). Metabolites of $\mathrm{PGD}_{2}$ can transactivate members of the PPAR family of nuclear hormone receptors. B. Epidermal growth factor receptor (EGFR), aberrantly expressed in prostate carcinoma, can activate cell growth through the RTK/PI3K/AKT pathway. COX-2 inhibitors induce apoptosis by blocking AKT activation in prostate cancer cells independently of $\mathrm{Bcl}-2$. Otherwise, overexpression of Her2/neu has been implicated in the activation of the AR and in inducing androgen-resistant prostate cancer cell growth. $\mathbf{C}$ NSAIDs can decrease the levels of the anti-apoptotic gene bcl-xL, thereby increasing the cellular ratio of $\mathrm{BAX} / \mathrm{bcl}-\mathrm{xL}$. There is also evidence that micro-tubule-interfering agents stimulate COX-2 transcription; otherwise, microtubule-interfering agents inhibit Bcl-2 activity by inducing Bcl-2 phosphorylation
\end{abstract}

There is also recent evidence that microtubule-interfering agents, including taxanes, stimulate COX-2 transcription [61]. This could modify the efficacy of this class of drugs. Thus, coadministration of a selective COX-2 inhibitor with cytotoxic drugs, such as taxanes, might enhance their anti-cancer activity. Induced expression of $\mathrm{COX}-2$ is at least partly responsible for the increased resistance to chemotherapeutic drugs. Furthermore, systemic inhibition of COX-2 activity may abrogate this resistance, thus opening a new avenue for increasing the efficacy of antitumour drugs against hormone refractory prostate cancer (HRPC). Activation of prosurvival pathways mediated through up-regulation of inducible enzyme COX-2 due to inflammation or stress appears to be one of the limiting factors in reducing the efficacy of chemodrugs and increasing metastatic potentials of established tumours. A taxane-containing regimen will be selected for clinical study, because prior work [61] found that this chemotherapeutic agent induced COX-2 and prostaglandin biosynthesis. A selective COX-2 inhibitor would be expected to prevent the possible negative action of this chemotherapeutic agent. Modulation of the $\mathrm{Bcl}-2$ pathway may also contribute to the antitumour effects noted with the nonsteroidal antiinflammatory drugs and COX-2-specific inhibitors [62]. Several agents with preclinical activity in prostate cancer have now been demonstrated to modulate $\mathrm{Bcl}-2$. The taxanes inhibit $\mathrm{Bcl}-2$ activity by inducing Bcl-2 phosphorylation.

Cytotoxicity of single or combination treatment in- creased apoptosis, which was associated with caspase-3 and -9 activation, increased BAD protein, but decreased protein levels of X-linked inhibitor of apoptosis protein (XIAP) and Bcl-xL. Overall, a synergistic enhancement of antitumour activity in combination treatment was observed in vitro and an additive effect in vivo [63]. These results indicate that a combination of cytotoxic drug as taxanes with COX-2 inhibitor has a synergistic effect and therefore improves the cytotoxicity of either drug (Fig. 4).

Furthermore, increased resistance to cytotoxic anti- tumour drugs is also known to be dependent on associated stromal cells in many tumours. Data presented recently [63] demonstrate that stromal factor induced overexpression of COX-2 and thereby increased release of cytoprotective prostacyclin $\mathrm{PGI}_{2}$ in prostate carcinoma cells, and may be one of the factors responsible for limiting the efficacy of chemodrugs on prostate carcinoma cells. Stromal cells and factors derived from them have been known to influence therapeutic response in vivo and reduce cytotoxicity in vitro in a variety of tumours and cell types [64]. Some reports document that stromal cells alter expression of cell adhesion molecules, integrins, cytokines, chemokines and also cell cycle-related events. In fact it was demonstrated that 
coculture of prostate carcinoma cells ( $\mathrm{LNCaP}$ ) with osteoblasts cells caused an increase in the number of prostate cells in $\mathrm{G}_{0}-\mathrm{G}_{1}$ and a decrease in $S$ and $\mathrm{G}_{2}-\mathrm{M}$ phases of cell cycle. This stromal-induced enhanced $\mathrm{G}_{0}-\mathrm{G}_{1}$ checkpoint control affected the chemosensitivity of prostate cancer cell line to chemodrugs [65].

In the other regard, the EGF receptor is frequently aberrantly expressed in prostate carcinoma [64] and has been proposed as a target for treatment of prostate carcinoma [66, 67]. The EGF/EGFR transduction pathway has been recognised to play a crucial role in metastatic prostate cancer cells [68, 69]. EGFR and its ligands EGF/TGF $\alpha$ appear to participate through autocrine and paracrine loops in prostatic tumour growth invasiveness and angiogenic processes by activating MAPK/PI3K and PLCg intracellular cascades and so increasing the cellular expression of PKA signalling [70, 71]. The activation of EGFR might lead to the negative modulation of apoptotic signals by activating PI3K/AKT survival cascade and inhibiting ceramide accumulation [72]. In vitro, simultaneous EGFR inhibitor and nitric oxide re- leasing compound might lead to a synergy in the ceramide and reactive oxygen species production, which might cause cellular membrane damage of metastatic prostate carcinoma cells [73]. Clinical benefits might derive from a regimen that combined a COX-2 inhibitor with an EGF receptor inhibitor (Fig. 4). COX-2 and EGFR are gaining significant attention as potential chemopreventive agents [77]. Targeting COX-2 and EGFR separately has shown promising antitumour activity. As both COX-2 and EGFR are involved in prostate carcinogenesis, COX-2 and EGFR inhibitors can be used as therapeutic strategies to treat prostate carcinoma; combinations of both inhibitors have been reported to show synergistic additive effects in preclinical studies [78].

The current trials advance prior preclinical work that implicates synergistic effects of combining COX inhibitors with cytotoxic chemotherapy and/or pharmacologic agents. In this way, at cellular levels via COX-2 independent mechanisms, celecoxib are capable of inducing cell cycle arrest at the $\mathrm{G}_{1}-\mathrm{S}$-phase transition [74], perturbing intracellular calcium by inhibiting endoplasmic reticulum $\mathrm{Ca}^{2+}$-ATPases [75], inhibiting cancer cell growth and mediating protein-protein interactions between heat shock protein 70 (HSP70) and p53 at low doses [76]. Recently, there has been evidence of modest biologic activity for celecoxib in men with rising PSA levels after radical prostatectomy and/or radiation therapy for prostate cancer. A recent study suggests that celecoxib with weekly docetaxel and estramustine is an effective and safe treatment for patients with hormone-refractory prostate cancer, but it does not seem to add any benefit to docetaxel [79].

\section{Conclusions}

One of the first clues that cyclooxygenase inhibition might be an effective approach to preventing cancer came from epidemiological studies. Preclinical and clinical evidence has established COX-2 as an attractive therapeutic or chemopreventive target in the prostate. Inducible COX-2 affects synthesis of prostaglandins from AA and is frequently activated during inflammation and carcinogenesis. COX-2 is also overexpressed in preneoplastic prostate lesions and inducible prostaglandin synthase is overexpressed in prostate carcinoma. It is also notable that epidemiologic data demonstrated a role for aspirin in suppressing prostate carcinogenesis.

Taken together, these findings clearly indicate that chronic elevation of COX-2 is pathological and suggest that inhibition of COX-2 via pharmacological means or regulation of its expression can limit the development or progression of human prostate cancer.

The investigations should be commended for designing and conducting trials which are excellent examples of translational research, by addressing whether NSAIDs can be administered in combination with chemotherapy. Undoubtedly the potential use of selective COX-2 inhibitors for the treatment of prostate cancer will also be investigated.

The ultimate goal of cancer treatment is to specifically prevent the growth of precancerous or cancerous cells without affecting normal cells. This is particularly important in chemoprevention and treatment of early disease, which typically involves long-term treatment of healthy subjects. 
Acknowledgements

We are grateful to Ana Carro Ramos for typing the manuscript. Guadalupe Aparicio Gallego is supported by Fundación Juan Canalejo Marítimo de Oza from A Coruña (Spain) and Silvia Díaz Prado is a beneficiary of an Isidro Parga Pondal contract by Xunta de Galicia (Spain).

\section{References}

1. Tremblay C, Dore M, Bochsler PN, Sirois J (1999) Induction of prostaglandin G/H synthase in a canine model of spontaneous prostatic adenocarcinoma. J Natl Cancer Inst 91:1398-1403

2. Gupta S, Srivastava M, Ahmad N et al (2000) Overexpression of cyclooxygenase-2 in human prostate adenocarcinoma. Prostate 42:73-79

3. Kirschenbaum A, Klausner AP, Lee R et al (2000) Expression of cyclooxygenase-1 and cyclooxygenase- 2 in the human prostate. Urology 56:671-676

4. Madaan S, Abel PD, Chaudhary DS et al (2000) Cytoplasmic induction and over-expression of cyclooxygenase-2 in human prostate cancer: implications for prevention and treatment. BJU Int 86:736-741

5. Tanji N, Kikugawa T, Yokoyama M (2000) Immunohistochemical study of cyclooxygenases in prostatic adenocarcinoma; relationship to apoptosis and Bcl-2 protein expression. Anti- cancer Res 20:2313-2319

6. Zha S, Gage WR, Sauvageot J et al (2001) Cyclooxygenase-2 is upregulated in proliferative inflammatory atrophy of the prostate, but not in prostate, but not in prostate carcinoma. Cancer Res 61:8617-8623

7. Shappell SB, Manning S, Boeglin WE et al (2001) Alterations in lipoxygenase and cy- clooxygenase-2 catalytic and mRNA expression in prostate carcinoma. Neoplasia 3:287-303

8. Nie D, Hillman GG, Geddes T et al (1998) Platelet-type 12-lipoxyenase in a human prostate carcinoma stimulates angiogenesis and tumor growth. Cancer Res 58:4047-4051

9. Timar J, Raso E, Dome B et al (2000) Expression, subcellular localization and putative function of platelet-type 12-lipoxygenase in human prostate cancer cell lines of different metastatic potential. Int J Cancer 87:37-43

10. Tjandrawinata RR, Dahya R, Hughes-Fulford M (1997) Induction of cyclo-oxygenase-2 mRNA by prostaglandin E2 in human prostatic carcinoma cells. Br J Cancer 75:1111-1118

11. Rose DP, Connolly JM (1991) Effects of fatty acids and eicosanoid synthesis inhibitors on the growth of two human prostate cancer cell lines. Prostate 18:243-254

12. Nolan RD, Danilowicz RM, Eling TE (1988) Role of arachidonic acid metabolism in the mitogenic response of BALB/c 3T3 fibroblasts to epidermal growth factor. Mol Pharmacol 33: 650-656

13. Glasgow WC, Wling TE (1989) Involvement of linoleic acid metabolites in epidermal growth factor-stimulated mitogenesis in BALB/c-3T3 fibroblast. Proc Am Assoc Cancer Res 30:102

14. Shaul PW, Anderson RG (1998) Role of plasmalemmal caveolae in signal transduction. Am J Physiol 275:L843L851

15. Zhuang L, Lin J, Lu ML et al (2002) Cholesterol- rich lipid rafts mediate akt-regulated survival in prostate cancer cells. Cancer Res 62: 2227-2231

16. Yoshimura R, Sano H, Masuda C et al (2000) Expression of cyclooxygenase-2 in prostate car- cinoma. Cancer 89:589-596

17. Gao X, Grignon DJ, Chbihi T et al (1995) Elevated 12-lipoxygenase mRNA expression correlates with advanced stage and poor differentiation of human prostate cancer. Urology 46:227-237

18. Kinzler KW, Vogelstein B (1996) Lessons from hereditary colorectal cancer. Cell 87:159-170

19. Honn KV, Tang DG, Gao X et al (1994) 12-Li- poxygenases and 12(S)-HETE in cancer metastasis. Cancer Metastasis Rev 13:365-396

20. Eberhart CE, Coffey RJ, Radhika A et al (1994) Up-regulation of cyclooxygenase 2 gene expression in human colorectal adenomas and adenocarcinomas. Gastroenterology 107:1183-1188

21. Sano H, Kawahito Y, Wilder RL et al (1995) Expression of cyclooxygenase -1 and -2 in human colorectal cancer. Cancer Res 55:3785-3789

22. Chan TA, Morin PJ, Vogelstein B, Kinzler KW (1998) Mechanisms underlying nonsteroidal an- tiinflammatory drug-mediated apoptosis. Proc Natl Acad Sci U S A 95:681-686

23. Sheng H, Shao J, Morrow JD et al (1998) Modulation of apoptosis and Bcl-2 expression by prostaglandin E2 in human colon cancer cells. Cancer Res 58:362-366

24. Liu XH, Yao S, Kirschenbaum A, Levine AC (1998) NS398, a selective cyclooxygenase-2 inhibitor, induces apoptosis and down-regulates bcl-2 expression in LNCaP cells. Cancer Res 58:4245-4249

25. Hsu AL, Ching TT, Wang DS et al (2000) The cyclooxygenase-2 inhibitor celecoxib induces apoptosis by blocking Akt activation in human prostate cancer cells independently of Bcl-2. J Biol Chem 275:11397-11403

26. Johnson AJ, Song X, Hsu A, Chen C (2001) Apoptosis signalling pathways mediated by cy- clooxygenase-2 inhibitors in prostate cancer cell. Adv Enzyme Regul 41:221-235

27. Song X, Lin HP, Johnson AJ et al (2002) Cyclooxygenase-2 player or spectator in cyclooxygenase-2 inhibitorinduced apoptosis in prostate cancer cells. J Natl Cancer Inst 94: 585-591

28. Zhu J, Song X, Lin HP et al (2002) Using cy- clooxygenase-2 inhibitors as molecular plat- forms to develop a new class of apoptosis-inducing agents. J Natl Cancer Inst 94:1745-1757 
29. Kulp SK, Yang YT, Hung CC et al (2004) 3- Phosphoinositide-dependent protein kinase- 1/Akt signalling represents a major cyclooxygenase-2-independent target for celecoxib in prostate cancer cells. Cancer Res 64:1444-1451

30. Patel MI, Subbaramaiah K, Du B et al (2005) Celecoxib inhibits prostate cancer growth: evidence of a cyclooxygenase-2-independent mechanism. Clin Cancer Res 11:1999-2001

31. Narayanan BA, Narayanan NK, Pttman B, Reddy BS (2006) Adenocarcinoma of the mouse growth inhibition by celecoxib: down regulation of transcription factors involved in COX-2 inhibition. Prostate 66:257-265

32. Sweeney C, Li L, Shanmugam R et al (2004) Nuclear factor/kappaB is constitutively activated in prostate cancer in vitro and is over expressed in prostatic intraepithelial neoplasia and adenocarcinoma of the prostate. Clin Cancer Res 10:5501-5507

33. Pan Y, Zhang JS, Gazi MH, Young CYF (2003) The cyclooxygenase 2-specific nonsteroidal anti-inflammatory drugs celecoxib and nimesulide inhibit androgen receptor activity via induction of c-Jun in prostate cancer cells. Cancer Epidemiol Biomarkers Prev 12:769-774

34. Murtha PE, Zhu W, Zhang J et al (1997) Effects of Ca++ mobilization on expression of androgen-regulated genes: interference with androgen receptor-mediated transactivation by AP-I proteins. Prostate 33:264-270

35. Schule R, Rangarajan P, Kliewer S et al (1990) c-Jun and the glucocorticoid receptor. Cell 62:1217-1226

36. Sato N, Sadar MD, Bruchovsky N et al (1997) Androgenic induction of prostate-specific anti- gen gene is repressed by protein-protein inter- action between the androgen receptor and AP- $1 / \mathrm{c}-\mathrm{Jun}$ in the human prostate cancer cell line LNCaP. J Biol Chem 272:17485-17494

37. Fronsdal K, Engedal N, Slagsvold T, Saatcioglu F (1998) CREB binding protein is a coactivator for the androgen receptor and mediates cross-talk with AP-1. [erratum appears in J Biol Chem 1999; 274:25188.] J Biol Chem 273:31853-31859

38. Wagner EF (2001) AP-1: introductory remarks. Oncogene 20:2334-2335

39. Steinbach G, Lynch PM, Phillips RK et al (2000) The effect of celecoxib a cycloxygenase- 2 inhibitor, in familial adenomatous polyposis. N Engl J Med 342:1946-1952

40. Howe LR, Subbaramaiah K, Brown AM, Dannenberg AJ (2001) Cyclooxygenase-2: a target for the prevention and treatment of breast cancer. Endocr Relat Cancer 8:97-114

41. Uotila P, Valve E, Martikainen P et al (2001) Increased expression of cyclooxygenase-2 and nitric oxide synthase-2 in human prostate cancer. Urol Res 29:23-28

42. Zha S, Gage WR, Sauwageot J et al (2001) Cyclooxygenase-2 is up-regulated in proliferative inflammatory atrophy of the prostate, but not in prostate carcinoma. Cancer Res 61:8617-8623

43. Shappell SB, Manning S, Boeglin WE et al (2001) Alterations in lipoxygenase and cyclooxy- genase-2 catalytic activity and mRNA expression in prostate carcinoma. Neoplasia 3:287-303

44. Kamijo T, Sato T, Nagatomi Y, Kitamura T (2001) Induction of apoptosis by cyclooxyge- nase- 2 inhibitors in prostate cancer cell lines. Int J Urol 8:S35-S39

45. Liu XH, Kirschenbaun A, Yao S et al (2000) Inhibition of cyclooxygenase-2 suppresses an- giogenesis and the growth of prostate cancer in vivo. J Urol 164:820-825

46. Norrish AE, Jackson RT, McRae CU (1998) Non- steroidal anti-inflammatory drugs and prostate cancer progression. Int J Cancer 77: 1322-1327

47. Paganini-Hill A, Chao A, Ross RK, Henderson BE (1989) Aspirin use and chronic diseases: a co- hort study of the elderly. Br Med J 299:1247-1250

48. Thun MJ, Namboodiri MM, Calle EE et al (1993) Aspirin use and risk of fatal cancer. Cancer Res 53:1322-1327

49. Rose P, Connolly JM (1991) Effects of fatty acids and eicosanoid synthesis inhibitors on the growth of two human prostate cancer cell lines. Prostate 18:243-254

50. Chaudry AA, Wahle KWJ, McClinton S, Moffat LEF (1994) Arachidonic acid metabolism in benign and malignant prostatic tissue in vitro: effects of fatty acids and cyclooxygenase inhibitors. Int J Cancer 57:176-180

51. Viljoen TC, van Aswegen CH, du Plessis DJ (1995) Influence of acetylsalicylic acid and metabolites on DUᄀ-145 prostatic cancer cell proliferation. Oncology 52:465-469

52. Tjandrawinata RR, Dahiya R, Hughes-Fulford M (1997) Induction of cyclo-oxygenase-2 mRNA by prostaglandin E2 in human prostatic carcinoma cells. Br J Cancer 75:1111-1118

53. Goluboff ET, Prager D, Rukstalis D et al (2001) Safety and efficacy of exisulind for treatment of recurrent prostate cancer after radical prostatectomy. J Urol 166:882-886

54. Ridgeon GH, Kandonz M, Meram A, Honn KV (2002) Mechanisms controlling cell cycle arrest and induction of apoptosis after 12-lipoxygenase inhibition in prostate cancer cells. Cancer Res 62:2721-2727

55. Yamamoto K, Arakawa T, Ueda N, Yamamoto S (1995) Transcriptional roles of nuclear factor $\kappa$ B and nuclear factor-interleukin-6 in the tumor necrosis factor $\alpha$-dependent induction of cyclooxygenase-2 in MC3T3-E1 cells. J Biol Chem 270:31315-31320

56. Yamamoto Y, Yin MJ, Lin KM, Gaynor RB (1999) Sulindac inhibits activation of the NF- $\kappa$ B pathway. J Biol Chem 274:27307-27314

57. Lehmann JM, Lenhard JM, Oliver BB et al (1997) Peroxisome proliferator-activated receptors alpha and gamma are activated by indomethacin and other non-steroidal anti-inflammatory drugs. J Biol Chem 272:3406-3410

58. Keller H, Dreyer C, Mdin J et al (1993) Fatty acids and retinoids control lipid metabolism through activation of peroxisome proliferators-activated receptor-retinoid X receptor heterodimers. Proc Natl Acad Sci USA 90:2160 2164

59. Forman BM, Chen J, Evans RM (1997) Hypolipidemic drugs, polyunsaturated fatty acids, and eicosanoids are ligand for peroxisome proliferators-activated receptors $\alpha$ and $\gamma$. Proc Natl Acad Sci U S A 94:4312-4317 
60. Kliewer SA, Sundseth SS, Jones SA et al (1997) Fatty acids and eicosanoids regulate gene expression through direct interactions with peroxisome proliferator-activated receptors $\alpha$ and $\gamma$. Proc Natl Acad Sci U S A 94:43184323

61. Subbaramiah D, Hart JC, Norton L, Dannenberg AJ (2000) Microtubule-interfering agents stimulate the transcription of cyclooxygenase-2. Evidence for involvement of ERK1/2 and p38 mitogen-activated protein kinase pathways. J Biol Chem 275:14838-14845

62. Liu QY, Stein CA (1997) Taxol and estramustine-induced modulation of human prostate cancer cell apoptosis via alteration in bcl-xL and bak expression. Clin Cancer Res 3:2039-2046

63. Dandekar DS, Lopez M, Carey RI, Lokeshwas BL (2005) Cyclooxygenase-2 inhibitor celecoxib augments chemotherapeutic drug-induced apoptosis by enhancing activation of caspase- 3 and -9 in prostate cancer cells. Int J Cancer 115:484-492

64. Hadlehurst LA, Landowski TH, Dalton WS (2003) Role of the tumor microenvironment in mediating de novo resistance to drugs and physiological mediators of cell death. Oncogene 22:7396-7402

65. Pinski J, Parikh A, Bova GS, Isaacs JT (2001) Therapeutic implications of enhanced G(0)/ G(1) checkpoint control induced by cocultured of prostate cancer cells with osteoblasts. Cancer Res 61:6372-6376

66. Scher HI, Sarkis A, Reuter V et al (1995) Changing pattern of expression of the epidermal growth factor receptor and transforming growth factor alpha in the progression of pro- static neoplasms. Clin Cancer Res 1:545-550

67. Morris MJ, Reuter VE, Kelly WK et al (2002) HER-2 profiling and targeting in prostate carcinoma. Cancer 94:980-986

68. Mimeault M, Pommery N, Henichart JP (2003) New advances on prostate carcinogenesis and therapies: involvement of EGF-EGFR transduc- tion system. Growth Factors 21:1-14

69. Kim HG, Kassis J, Souto JC et al (1999) EGF receptor signaling in prostate morphogenesis and tumorigenesis. Histol Histopathol 14:1175-1182

70. Putz T, Culig Z, Eder IE et al (1999) Epidermal growth factor (EGF) receptor blockade inhibits the action of EGF, insulin-like growth factor I, and a protein kinase A activator on the mitogen- activated protein kinase pathway in prostate cancer cell lines. Cancer Res 59:227-233

71. Chen Y, Hughes-Fulford M (2000) Prostaglandin E2 and the protein kinase A pathway mediate arachidonic acid induction of c-fos in human prostate cells. Br J Cancer 82:2000-2006

72. Coroneos E, Martinez M, McKenna S, Kester N (1995) Differential regulation of sphingomyelinase and ceramidase activities by growth factors and cytokines. Implications for cellular proliferation and differentiation. J Biol Chem 270:23305-23309

73. Mimeault N, Jouy N, Depreux P, Henichart JP (2005) Synergistic antiproliferative and apoptotic effects induced by mixed epidermal growth factor receptor inhibitor ZD1839 and nitric oxide donor in human prostatic cancer cell lines. Prostate 62:187-199

74. Narayanan NK, Condon MS, Bosland MC et al (2003) Suppression of N-methyl-N-nitrosourea /testosteroneinduced rat prostate cancer growth by celecoxib: effects on cyclooxygenase- 2 cell cycle regulation, and apoptosis mechanisms (s). Clin Cancer Res 9:3503-3513

75. Johnson, AJ, Hsu AL, Lin HP et al (2002) The cyclooxygenase-2 inhibitor celecoxib perturbs intracellular calcium by inhibiting endoplasmic reticulum $\mathrm{Ca} 2+-$ ATPases: a plausible link with its anti-tumor effect and cardiovascular risks. Biochem J 366:831-837

76. Narayanan NK, Narayanan BA, Bosland M et al (2006) Docosahenoic acid in combination with celecoxib modulates HSP70 and p53 proteins in prostate cancer cells. Int J Cancer 119:1536-1598

77. Dannenberg AJ, Lippman SC, Mann JR et al (2005) Cyclooxygenase-2 and epidermal growth factor receptor: pharmacologic targets for chemoprevention. J Clin Oncol 23:254-266

78. Choe MS, Zhang X, Shin HJC et al (2005) Interaction between epidermal growth factor receptor-and cyclooxygenase 2-mediated pathways and its implications for the chemoprevention of head and neck cancer. Mol Cancer Ther 4:1448-1455

79. Carles J, Font A, Mellado B et al (2007) Weekly administration of docetaxel in combination with estramustine and celecoxib in patients with advanced hormone-refractory prostate cancer: final results from a phase II study. Br J Cancer 97:1206-1210 